TITLE:

\title{
A machining test to calibrate rotary axis error motions of five-axis machine tools and its application to thermal deformation test
}

\author{
$\operatorname{AUTHOR}(\mathrm{S})$ :
}

Ibaraki, Soichi; Ota, Yusuke

\section{CITATION:}

Ibaraki, Soichi ... [et al]. A machining test to calibrate rotary axis error motions of five-axis machine tools and its application to thermal deformation test. International Journal of Machine Tools and Manufacture 2014, 86: 81-88

\section{ISSUE DATE:}

2014-11

URL:

http://hdl.handle.net/2433/189418

\section{RIGHT:}

(c) 2014 Elsevier Ltd.; この論文は出版社版でありません。引用の際には 出版社版をご確認ご利用ください。; This is not the published version. Please cite only the published version. 


\title{
A machining test to calibrate rotary axis error motions of five-axis machine tools and its application to thermal deformation test
}

\begin{abstract}
This paper proposes a machining test to parameterize error motions, or position-dependent geometric errors, of rotary axes in a five-axis machine tool. At the given set of angular positions of rotary axes, a square-shaped step is machined by a straight end mill. By measuring geometric errors of the finished test piece, the position and the orientation of rotary axis average lines (location errors), as well as position-dependent geometric errors of rotary axes, can be numerically identified based on the machine's kinematic model. Furthermore, by consequently performing the proposed machining test, one can quantitatively observe how error motions of rotary axes change due to thermal deformation induced mainly by spindle rotation. Experimental demonstration is presented.

Keywords: Five-axis machine tool, machining test, error motion, location error, error calibration, kinematic model, thermal deformation.
\end{abstract}




\section{Introduction}

Machine tools with two rotary axes to tilt a tool and/or a workpiece, in addition to three orthogonal linear axes, are collectively called five-axis machine tools. On five-axis machine tools, error motions of each linear/rotary axis, as well as its alignment (assembly) errors, are accumulated in the positioning error of a tool relative to a workpiece. ISO/DIS 10791-1 [1], currently under a revision process in ISO/TC 39/SC 2, contains quasi-static tests to calibrate alignment errors (location errors) of rotary axes. ISO 230-7 [2] describes the tests to observe error motions of rotary axis (ISO 230-7 [2] mainly targets a spindle but can be in principle applied to any rotary axes). Such a "direct" measurement [3] requires a different setup for each error, and thus full evaluation often takes significant time.

For more efficient error calibration, many "indirect" measurement methodologies, where each alignment error or error motion is indirectly identified from a set of tool center point (TCP) profiles measured with respect to the work table, have been studied. A comprehensive review can be found in $[3,4]$. The application of the ball bar test to dynamic measurement for rotary axes has been studied by many researchers $[5,6,7]$ and is now included in ISO/FDIS 10791-6 [8], also currently under a revision process in ISO/TC 39/SC 2. The R-test [9, 10] is also in ISO/FDIS 10791-6. The static version of analogous "chase-the-ball" test [10] can be done by using a touch-triggered probe $[11,12]$. Commercial probe-based calibration systems are now available from several vendors, e.g. Renishaw [13].

Although it is important to evaluate geometric errors of rotary axes by such a non-cutting measurement, typical machine tool users consider more 
the machine's accuracy when it performs actual machining. Non-cutting tests are sometimes performed when the machine is "cold." Although the accuracy test standards, e.g. [1, 8], strongly suggest performing sufficient machine warm-up before tests, the machine's thermal condition may not be exactly the same as actual machining conditions, since the spindle stops in measuring cycles. In "normal" operating condition with spindle rotation, the machine's geometric errors may be significantly different from those in "cold" condition.

The heat generated by spindle rotation most typically displaces the TCP to the Z-direction. In the three-axis machining, such a simple translational error may not cause significant geometric error of the machined workpiece, as long as the deformation does not vary much throughout the machining process. In five-axis machining, even such a constant expansion changes the position of rotary axes with respect to the machine coordinate system, which likely results in the machined workpiece's geometric errors. ISO 230-3 [14], describing thermal tests for machine tools, only investigates thermal influence on the positioning by linear axes. No test is specified for five-axis machine tools. The importance of thermal tests for rotary axes has been discussed only lately in the literature. Recent works include the application of the R-test $[15,16,17]$.

A machining test can evaluate the machine's error motions when it performs the machining. NAS (National Aerospace Standard) 979 [18], Clause 4.3.3.8.1, describes a cone frustum five-axis machining test. Since it is only standard well known describing a five-axis machining test, it is widely accepted by many machine tool builders as one of final performance tests. 
ISO/TC 39/SC 2 is currently discussing its inclusion in the revision of ISO 10791-7 [19]. Although it gives a good demonstration of the machine's overall machining performance, it is generally difficult to diagnose error sources from the measured geometry of the finished test piece [20, 21]. Some five-axis machining tests have been proposed in the literature or the industry, e.g. the truncated square pyramid [22], a set of surfaces machined by a ball end mill at different angular positions [23] and the S-shaped thin wall [24]. None of them aims for the diagnosis of error causes from the geometry of the finished test piece.

The objective of this paper is to propose a new five-axis machining test such that geometric errors of rotary axes can be separately identified by evaluating the geometric error of the machined test piece. In [25], a part of the authors presented a machining test to identify position and orientation errors of the axis average line (location errors) of two rotary axes. More lately, a rather complex test was presented in [26] that enables the identification of rotary axis location errors. This paper presents a new machining test by extending [25] to position-dependent geometric errors, or error motions, of rotary axes. While location errors only represent "average" position and orientation errors of rotary axes, the proposed test fully parameterize how the position and the orientation of the axis of rotation change with its rotation.

Furthermore, the paper will present the observation of the machine's thermal stability by consequently performing the proposed machining test. Experimental demonstration will be presented. 


\section{Proposed machining test}

This paper considers a five-axis machine configuration with a titling rotary table (driven by B- and C-axes) depicted in Fig. 1. The swivel axis (B-axis) rotates over $-90 \sim+90^{\circ}$. In principle, the basic idea of this paper can be straightforwardly extended to any five-axis configurations.

The proposed machining test is illustrated in Fig. 2. At $B_{i}=C_{j}=0^{\circ}$, a square-shaped step is machined by a straight end mill with driving $\mathrm{X}$ - or Y-axis only (the reference step). Then, the square step is machined at different heights at $C_{j}=90,180,270^{\circ}$. This is repeated at every combination of $B_{i}=-90,0,90^{\circ}$ and $C_{j}=0,90,180,270^{\circ}$. Total $4 \times 3=12$ finish cuts are made. Figure 3 shows the nominal geometry of the finished test piece. The finishing condition must be properly chosen such that the influence of tool deflection or surface roughness becomes sufficiently small. It is recommended to repeat the finishing with zero radial depth of cut, i.e. "zero cut.".

Then, the finished test piece's geometry is measured preferably by using a coordinate measuring machine (CMM). Figure 4 shows an example set of probed points on the finished test piece in our experiments (Section 5). The measurement coordinate system is set up such that 1) its X-axis is aligned to the -Y side face of the reference step, 2) its $\mathrm{Y}$-axis is parallel to one of the bottom faces of the reference step, and 3) the (X,Y) position of its origin is at the center of the reference step, and its $\mathrm{Z}$ position is on the average plane of four bottom faces of the reference step. The measurement coordinate system is also shown in Fig. 4. 


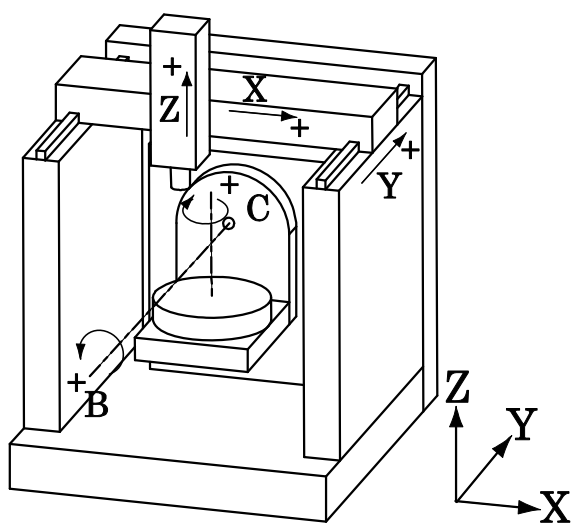

Figure 1: The configuration of the five-axis machine tool considered in this paper [33]. 


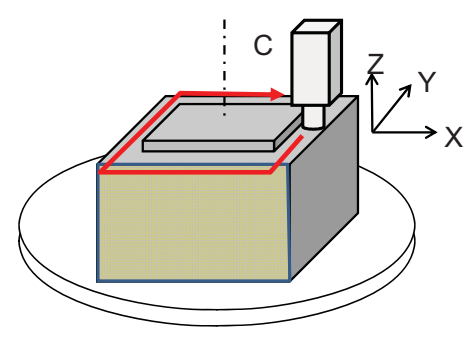

(a)

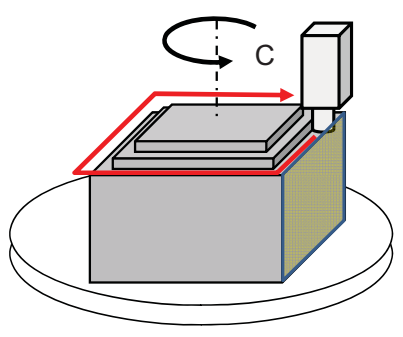

(b)

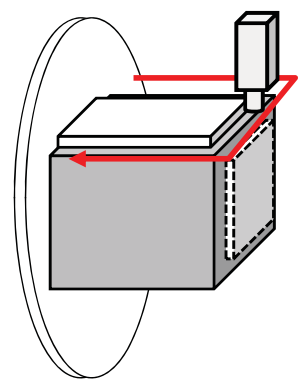

(c)

Figure 2: The proposed machining procedure; (a) Machine the reference square at $B=C=0^{\circ}$. (b) Rotate to $C=90^{\circ}$ and machine the same square. Repeat this at $C=180^{\circ}$ and $270^{\circ}$. (c) Rotate to $B=90^{\circ}$ and machine the same square. Repeat this at $C=0,90,180,270^{\circ}$. Repeat this at every combination of $B=-90,0,90^{\circ}$ and $C=0,90,180,270^{\circ}$. 


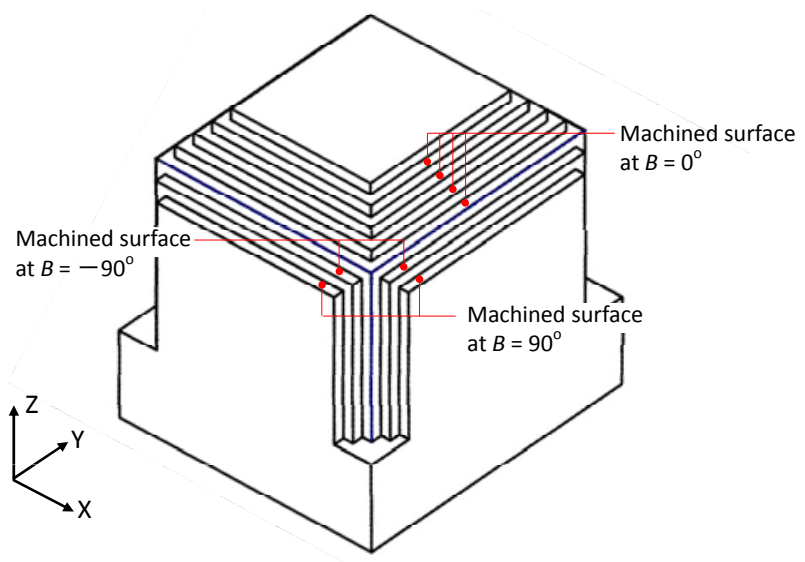

Figure 3: Finished test piece geometry.

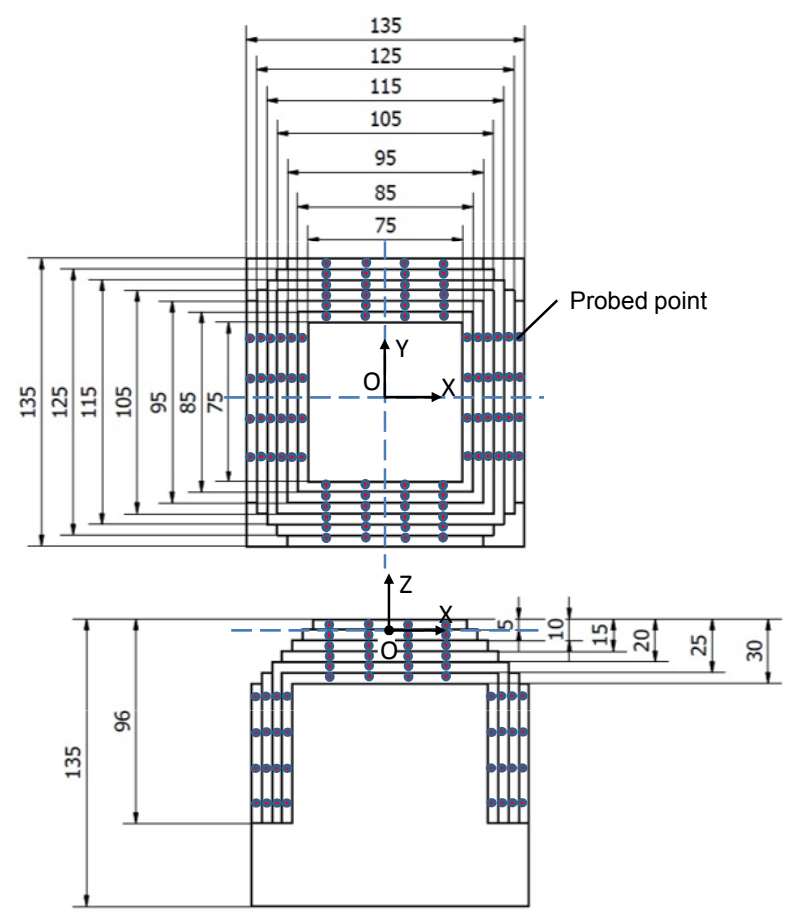

Figure 4: Finished test piece geometry and probed points in the experiment in Section 5 . 


\section{Identification of rotary axis geometric errors}

\subsection{Geometric error parameters to be identified}

In ISO 230-1 [27], the axis average line of a rotary axis is defined as "the straight line representing the mean location and orientation of its axis of rotation." Position and orientation errors of a rotary axis average line, called location errors in ISO 230-7 [2], are clearly among the most fundamental error factors in the five-axis kinematics. Table 1 shows location errors for the configuration in Fig. 1 [27, 28].

They only represent "average" position or orientation of a rotary axis. The axis of rotation may change its position and orientation with its rotation. Such an error motion can be parameterized by position-dependent geometric errors [20] shown in Table 2. They are defined such that:

$$
\frac{1}{N_{c}} \sum_{j=1}^{N_{c}} \delta y_{C B}\left(B_{i}, C_{j}\right)=\delta y_{C B}^{0}
$$

and analogous relationship holds for other parameters.

It is important to note that this paper assumes geometric errors of linear axes are sufficiently smaller than those of rotary axes. Many five-axis error calibration methodologies, briefly reviewed in Section 1, require synchronously driving rotary and linear axes, and it is therefore not possible in principle to separate error motions of rotary and linear axes. Linear axis error motions must be separately pre-calibrated and compensated by conventional measurement, e.g. ISO 10791-1 [1]. In practice, linear axis error motions are often major uncertainty contributors for the identification of rotary axis geometric errors. Their contribution will be studied in Section 6 . 
Table 1: Position and orientation errors of rotary axis average lines (location errors) for the machine configuration in Fig. 1. Correspondence to symbols depends on the machine tool coordinate system setup [27].

\begin{tabular}{l|l|l}
\hline Symbol $[27]$ & Symbol $[28]$ & Description \\
\hline$E_{X 0 B}$ & $\delta x_{B Y}^{0}$ & Position error of B-axis average line in X \\
$E_{Y 0 C}$ & $\delta y_{C B}^{0}$ & Position error of C-axis average line in Y \\
$E_{Z 0 B}$ & $\delta z_{B Y}^{0}$ & Position error of B-axis average line in Z \\
$E_{X(0 B) C}$ & $\delta x_{C B}^{0}$ & Position error of C-axis from B-axis in X \\
$E_{A 0 B}$ & $\alpha_{B Y}^{0}$ & Orientation error of B-axis avg line around X \\
$E_{B 0 B}$ & $\beta_{C B}^{0}$ & Initial angular positioning error of B-axis \\
$E_{C 0 B}$ & $\gamma_{B Y}^{0}$ & Orientation error of B-axis avg line around Z \\
$E_{A(0 B) C}$ & $\alpha_{C B}^{0}$ & Squareness error of C- to B-axis \\
\hline
\end{tabular}

Table 2: Position-dependent geometric errors (error motions) of rotary axes.

\begin{tabular}{l|l|l}
\hline Symbol $[27]$ & Symbol $[28]$ & Description \\
\hline$E_{X B}\left(B_{i}\right)$ & $\delta x_{B Y}\left(B_{i}\right)$ & Linear error motions of B-axis \\
$E_{Y B}\left(B_{i}\right)$ & $\delta y_{B Y}\left(B_{i}\right)$ & at $B=B_{i}$ in $\mathrm{X}, \mathrm{Y}$, and Z \\
$E_{Z B}\left(B_{i}\right)$ & $\delta z_{B Y}\left(B_{i}\right)$ & \\
$E_{A B}\left(B_{i}\right)$ & $\alpha_{B Y}\left(B_{i}\right)$ & Angular error motions of B-axis \\
$E_{B B}\left(B_{i}\right)$ & $\beta_{B Y}\left(B_{i}\right)$ & at $B=B_{i}$ around $\mathrm{X}, \mathrm{Y}$, and Z \\
$E_{C B}\left(B_{i}\right)$ & $\gamma_{B Y}\left(B_{i}\right)$ & \\
\hline$E_{X C}\left(B_{i}, C_{j}\right)-E_{X B}\left(B_{i}\right)$ & $\delta x_{C B}\left(B_{i}, C_{j}\right)$ & Linear error motions of C-axis \\
$E_{Y C}\left(B_{i}, C_{j}\right)-E_{Y B}\left(B_{i}\right)$ & $\delta y_{C B}\left(B_{i}, C_{j}\right)$ & at $B=B_{i}$ and $C=C_{j}$ in X, Y, and Z \\
$E_{Z C}\left(B_{i}, C_{j}\right)-E_{Z B}\left(B_{i}\right)$ & $\delta z_{C B}\left(B_{i}, C_{j}\right)$ & \\
$E_{A C}\left(B_{i}, C_{j}\right)-E_{A B}\left(B_{i}\right)$ & $\alpha_{C B}\left(B_{i}, C_{j}\right)$ & Angular error motions of B-axis \\
$E_{B C}\left(B_{i}, C_{j}\right)-E_{B B}\left(B_{i}\right)$ & $\beta_{C B}\left(B_{i}, C_{j}\right)$ & at $B=B_{i}$ and $C=C_{j}$ around X, Y, and Z \\
$E_{C C}\left(B_{i}, C_{j}\right)-E_{C B}\left(B_{i}\right)$ & $\gamma_{C B}\left(B_{i}, C_{j}\right)$ & \\
\hline
\end{tabular}




\subsection{Kinematic model}

The workpiece coordinate system is the coordinate system attached to the rotary table, and its origin is set at the nominal intersection of B- and Caxes. Suppose that the nominal TCP in the workpiece coordinate system is given by ${ }^{w} q^{*} \in \mathbb{R}^{3}$. The machine' $\mathrm{s}$ kinematic model, formulating the TCP position in the workpiece coordinate system when there exist rotary axis error motions in Table 2, plays an essential role in many "indirect" measurement approaches [3, 4], and has been long studied [29, 30, 6, 28]. Although it can be found in many previous publications, it is briefly reviewed here, since it is the basis for the identification algorithm to be presented in the following subsections.

When the B- and C-axes are nominally indexed at $B_{i}$ and $C_{j}$, the actual TCP position in the workpiece coordinate system, ${ }^{w} q \in \mathbb{R}^{3}$, under positiondependent geometric errors in Table 2, is given by:

$$
\left[\begin{array}{c}
{ }^{w} q \\
1
\end{array}\right]=\left({ }^{r} T_{w}\right)^{-1}{ }^{r} T_{w}^{*}\left[\begin{array}{c}
{ }^{w} q^{*} \\
1
\end{array}\right]
$$

where ${ }^{r} T_{w} \in \mathbb{R}^{4 \times 4}$ is the homogeneous transformation matrix (HTM) representing the transformation from the workpiece coordinate system to the machine coordinate system:

$$
\begin{aligned}
{ }^{r} T_{w}= & { }^{r} T_{b}^{b} T_{c} \\
{ }^{r} T_{b}= & D_{x}\left(\delta x_{B Y}\left(B_{i}\right)\right) D_{y}\left(\delta y_{B Y}\left(B_{i}\right)\right) D_{z}\left(\delta z_{B Y}\left(B_{i}\right)\right) \\
& D_{a}\left(\alpha_{B Y}\left(B_{i}\right)\right) D_{b}\left(\beta_{B Y}\left(B_{i}\right)\right) D_{c}\left(\gamma_{B Y}\left(B_{i}\right)\right) D_{b}\left(-B_{i}\right) \\
{ }^{b} T_{c}= & D_{x}\left(\delta x_{C B}\left(B_{i}, C_{j}\right)\right) D_{y}\left(\delta y_{C B}\left(B_{i}, C_{j}\right)\right) D_{z}\left(\delta z_{C B}\left(B_{i}, C_{j}\right)\right) \\
& D_{a}\left(\alpha_{C B}\left(B_{i}, C_{j}\right)\right) D_{b}\left(\beta_{C B}\left(B_{i}, C_{j}\right)\right) D_{c}\left(\gamma_{C B}\left(B_{i}, C_{j}\right)\right) D_{c}\left(-C_{j}\right)
\end{aligned}
$$


where $D_{*}(*) \in \mathbb{R}^{4 \times 4}$ denotes the HTM representing the translation in X, Y or Z and the rotation around X, Y, or Z (see e.g. [6] for their formulation). ${ }^{w} T_{r}^{*} \in \mathbb{R}^{4 \times 4}$ represents the nominal transformation, i.e.

$$
{ }^{r} T_{w}^{*}=D_{b}\left(-B_{i}\right) D_{c}\left(-C_{j}\right)
$$

In [31], a part of the authors furthermore presented the linearization of the model (3) as:

$$
\left[\begin{array}{c}
{ }^{w} q \\
1
\end{array}\right]=D_{x}(\Delta X) D_{y}(\Delta Y) D_{z}(\Delta Z) D_{a}(\Delta A) D_{b}(\Delta B) D_{c}(\Delta C)\left[\begin{array}{c}
{ }^{w} q^{*} \\
1
\end{array}\right]
$$

where

$\Delta X=-\left(\delta x_{B Y} \cos B_{i}+\delta z_{B Y} \sin B_{i}+\delta x_{C B}\right) \cos C_{j}+\left(\delta y_{B Y}+\delta y_{C B}\right) \sin C_{j}$

$\Delta Y=-\left(\delta x_{B Y} \cos B_{i}+\delta z_{B Y} \sin B_{i}+\delta x_{C B}\right) \sin C_{j}-\left(\delta y_{B Y}+\delta y_{C B}\right) \cos C_{j}$

$\Delta Z=\delta x_{B Y} \sin B_{i}-\delta z_{B Y} \cos B_{i}-\delta z_{C B}$

$\Delta A=-\left(\alpha_{B Y} \cos B_{i}+\gamma_{B Y} \sin B_{i}+\alpha_{C B}\right) \cos C_{j}+\left(\beta_{B Y}+\beta_{C B}\right) \sin C_{j}$

$\Delta B=-\left(\alpha_{B Y} \cos B_{i}+\gamma_{B Y} \sin B_{i}+\alpha_{C B}\right) \sin C_{j}-\left(\beta_{B Y}+\beta_{C B}\right) \cos C_{j}$

$\Delta C=\alpha_{B Y} \sin B_{i}-\gamma_{B Y} \cos B_{i}-\gamma_{C B}$

For the simplicity of notation, $\left(B_{i}\right)$ or $\left(B_{i}, C_{j}\right)$ with each geometric error parameter is omitted here. With the small angle approximation, Eq. (5) describes the linear relationship between each geometric error and the positioning error at the TCP in the workpiece coordinate system.

\subsection{Calculation of position and orientation of each step}

For each square-shaped step machined at $B_{i}$ and $C_{j}$, denote the $k$-th measured position in the measurement coordinate system by $p(i, j, k) \in \mathbb{R}^{3}$ 
$(k=1, \cdots N(i, j))$, where $N(i, j) \in \mathbb{R}$ is the number of probed points on the $(i, j)$ step. Suppose that its nominal position is given by $p^{*}(i, j, k) \in \mathbb{R}^{3}$. This and the following subsections present an algorithm is to identify positiondependent geometric errors in Table 2, as well as location errors in Table 1, from a set of $p(i, j, k)\left(i=1, \cdots N_{b}, j=1, \cdots N_{c}, k=1, \cdots, N(i, j)\right)$.

Denote the displacement of the $(i, j)$ step from its nominal position by $(\Delta x(i, j), \Delta y(i, j), \Delta z(i, j))$ in $\mathrm{X}, \mathrm{Y}$, and $\mathrm{Z}$ directions. Denote its orientation error by $(\Delta a(i, j), \Delta b(i, j), \Delta c(i, j))$ around $\mathrm{X}, \mathrm{Y}$, and $\mathrm{Z}$ axes. From a set of measured points, $p(i, j, k)$, they can be calculated by solving the following minimization problem:

$$
\min _{\Delta x(i, j), \cdots, \Delta c(i, j)} \sum_{k}\left\{\Delta p(i, j, k) \cdot n^{*}(i, j, k)\right\}^{2}
$$

where $n^{*}(i, j, k) \in \mathbb{R}^{3}$ is a unit vector representing the normal direction to the target surface. This term is needed since a touch-trigger probe is sensitive only to this direction.

$$
\Delta p(i, j, k)=p(i, j, k)-\hat{p}(i, j, k)
$$

and

$$
\begin{aligned}
{\left[\begin{array}{c}
\hat{p}(i, j, k) \\
1
\end{array}\right]=} & D(\Delta x(i, j)) D(\Delta y(i, j)) D(\Delta z(i, j)) \\
& D(\Delta a(i, j)) D(\Delta b(i, j)) c(\Delta c(i, j))\left[\begin{array}{c}
p^{*}(i, j, k) \\
1
\end{array}\right]
\end{aligned}
$$

By applying the small angle linear approximation, the problem (7) can be solved by the least square method. 


\subsection{Identification of rotary axis geometric errors}

In $[32,33]$, a part of the authors presented a probe-based approach to identify rotary axis geometric errors (see Section 4.2). In this algorithm, the position and the orientation of the test piece of the known geometry is measured in the machine coordinate system. In the present machining test, the geometry of the machined test piece is determined by the TCP trajectory in the workpiece coordinate system. This is an essential difference but their basic formulation is analogous.

Position/orientation errors of the $(i, j)$ step, $\Delta x(i, j), \cdots, \Delta c(i, j)$, calculated by solving Eq. (7), represent position/orientation errors of the TCP trajectory (a square path) in the workpiece coordinate system. Therefore, they should satisfy the kinematic relationship in Eq. (5). In other words, position-dependent geometric errors, given in in Table 2, can be obtained by solving Eq. (6) with $\Delta x(i, j)$ to $\Delta c(i, j)$ replacing the left-hand side of each equation.

An attention should be paid to the influence of rotary axis geometric errors on position/orientation of the measurement coordinate system. Recall that each step's position and orientation, represented by $\Delta x(i, j)$ to $\Delta c(i, j)$, are measured in reference to those of the reference step, machined at $B_{1}=C_{1}=0^{\circ}$. In other words, $\Delta x(i, j)$ to $\Delta c(i, j)$ must be zero at $B_{1}=C_{1}=0^{\circ}$. With this constraint, the first equation in Eq. (6) should be modified as:

$$
\begin{aligned}
& \Delta x(i, j)=-\left(\delta x_{B Y} \cos B_{i}+\delta z_{B Y} \sin B_{i}+\delta x_{C B}\right) \cos C_{j} \\
&+\left(\delta y_{B Y}+\delta y_{C B}\right) \sin C_{j}+\left(\delta x_{B Y}+\delta x_{C B}\right)
\end{aligned}
$$


$\Delta y(i, j)$ to $\Delta c(i, j)$ are formulated similarly. The B-axis position-dependent geometric errors, shown in Table 2, can be identified by using the least square method such that Eq. (10) (and analogously modified Eq. (6)) is best fit. The C-axis position-dependent geometric errors are given by solving same equations for each $B_{i}$ and $C_{j}$.

\section{Application to thermal test}

\subsection{Objective}

The thermal deformation is often among the most critical error contributors for machine tools. ISO 26303:2012 [34] describes the short-term capability test for machine tools, where a sample batch of test pieces is machined consequently and the statistical variation of geometric errors of test pieces is evaluated. Such a sequential machining test clarifies the machine's and the environment's thermal stability. Unlike the machining tests in [34], the present machining test enables a user to numerically observe how each geometric error changes with time. This section presents such an application of the proposed machining test.

\subsection{Comparison with non-cutting geometric error calibration}

First, we suggest performing one of non-cutting error calibration schemes, reviewed in Section 1, in the "cold" condition, i.e. before the machine reaches the thermal equilibrium representing the normal cutting condition by sufficient spindle warm-up. Then, by comparing with the present machining test, 
one can numerically observe the thermal influence of spindle rotation from the "cold" condition to the cutting condition.

In [33], a part of the authors presented a probe-based error calibration scheme illustrated in Fig. 5. At each $\left(B_{i}, C_{\mathrm{J}}\right)$, the part blank before the machining test is probed by using a touch-triggered probe. The number of probed points must be sufficient to calculate the test piece's position and orientation (Fig. 5 shows only an example). The Ref. [33] presented an algorithm to identify location errors and position-dependent geometric errors shown in Tables 1 and 2. It is important to note that the geometric inaccuracy of the test piece or their setup (alignment) error does not significantly affect the estimates of geometric error parameters. Therefore, the part blank of the unknown geometry can be used as the target, requiring no additional artefact such as a precision sphere, typically used in similar probe-based error calibration schemes, e.g. [13]. The surface roughness of the part blank should be small enough to ensure probing repeatability. Although this paper recommends the probe-based scheme in [33] for the convenience with the machining test, any other non-cutting calibration schemes, e.g. the R-test, can be employed.

\subsection{Proposed test procedure}

1. Mount the part blank on the machine table. Perform the probing procedure presented in [33] (Fig. 5).

2. Perform spindle warm-up till the thermal equilibrium is reached.

3. Rough cutting.

4. Finishing as described in Section 2. 


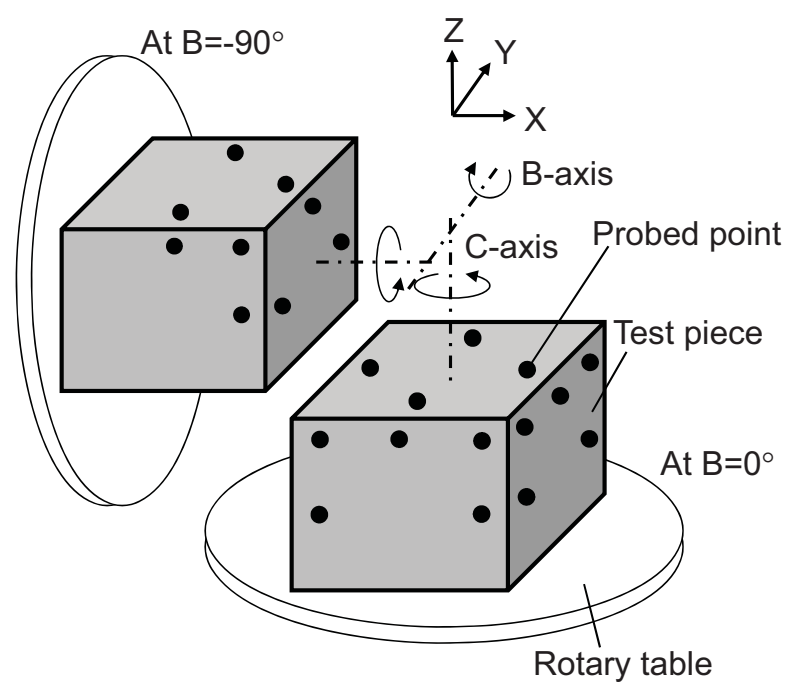

Figure 5: The probing procedure for the calibration fo rotary axis geometric errors presented in [33]. The position and the orientation of the test piece is measured at each combination of $B_{i}=-90,0,90^{\circ}$, and $C_{j}=0,90,180,270^{\circ}$.

5. Dismount the finished test piece. Repeat steps 3 and 4 to finish another test piece (in our experiment total three test pieces were finished).

6. The finished test pieces are measured as in Section 2.

\section{Experiment}

\subsection{Test setup}

The proposed machining test is conducted on a machining center of the configuration in Fig. 1. Table 3 shows major machining conditions. Figure 6 shows the machining test setup.

The pre-machining probing test was done by using a touch-triggered probe, OMP-400 by Renishaw (unidirectional repeatability: $0.35 \mu \mathrm{m}$ (max 
Table 3: Major machining conditions.

\begin{tabular}{l|l}
\hline Tool & Sintered carbide radius end mill, $\phi 8 \mathrm{~mm}, 3$ flutes \\
Workpiece material & Aluminum alloy JIS A5052 \\
Feed per tooth & $0.075 \mathrm{~mm} /$ tooth \\
Axial depth of cut & $5 \mathrm{~mm}$ \\
Radial depth of cut & $0 \mathrm{~mm}$ (zero cut) \\
Cutting speed & $100 \mathrm{~m} / \mathrm{min}$ \\
Cutting fluid & Air blow \\
Milling direction & Down cut \\
\hline
\end{tabular}

$2 \sigma$ value with $100 \mathrm{~mm}$ stylus), probe sphere: $\phi 6 \mathrm{~mm}$ (ruby)). The machining center's positioning resolution is $1 \mu \mathrm{m}$ on all the linear axes; the probe's measurement resolution is also $1 \mu \mathrm{m}$. The probe's direction-dependent pretravel variation was measured and compensated by the standard procedure suggested by the vendor.

The nominal geometry of the finished test piece is shown in Fig. 4. After the probing test (Step 1 in Section 4.3), the spindle warm-up was done for about $30 \mathrm{~min}$. The roughing and the finishing respectively took about 25 min. Total three test pieces were machined consequently.

Although it is preferable to measure the geometry of the finished test pieces by a CMM, in this experiment it is measured on-the-machine by using the same probe, assuming sufficiently high volumetric accuracy of the machine tool's linear axes. This measurement was done after the machine cools down sufficiently. With the machine' $\mathrm{s}$ B- and C-axes fixed at $B=C=0^{\circ}$, points shown in Fig. 4 are probed. Note that error motions of B- and C-axes do not influence this measurement. 


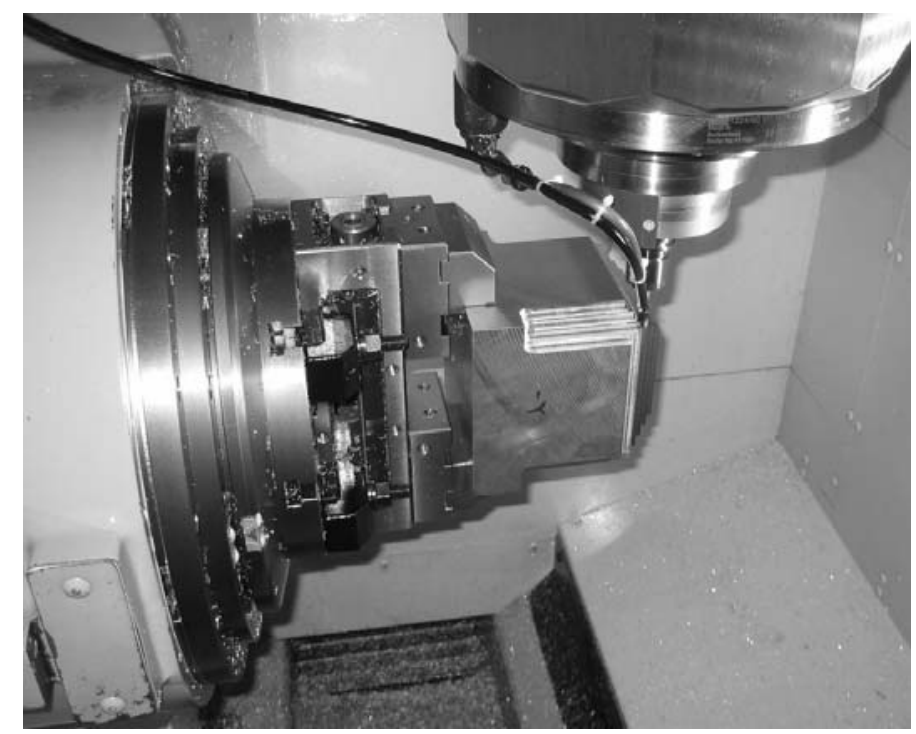

Figure 6: Machining test setup (at $B=-90^{\circ}$ ).

\subsection{Geometric error calibration by the first test piece}

First, the geometry of the first finished test piece was measured. Figure 7 shows the measured geometry of four steps on the first test piece's top face, each of which is machined at $B_{i}=0^{\circ}$, and $C_{j}=0,90,180,270^{\circ}$, respectively. In Fig. 7 , the $\operatorname{dots}(\bullet)$ represent the nominal probed position, $p^{*}(i, j, k)$, and the circles $(\bigcirc)$ represent the measured position, $p(i, j, k)$. The error between measured and nominal positions is magnified 1,000 times. The position and the orientation of each step, represented by $\Delta x(i, j) \sim \Delta c(i, j)$, are calculated by the algorithm in Section 3.3. In Fig. 7, the painted bold-line square represents calculated position and orientation of each step (those in Fig. 7(a) are calculated from probed points on side faces of each step, and those in Fig. 7(b) are calculated from probed points on the bottom face). Similarly, Fig. 8 shows the measured geometry of four steps on side faces, machined 
$B_{i}=90^{\circ}$, and $C_{j}=0,90,180,270^{\circ}$.

First, by such a graphical presentation of the finished test piece's geometric errors, one can make many intuitive observations on location errors or error motions of rotary axes, even without proceeding to their numerical parameterization. For example:

- In Fig. 7(a), the square machined at $C=-180^{\circ}$ is shifted by about +8 $\mu \mathrm{m}$ in $\mathrm{X}$-direction, and $+3 \mu \mathrm{m}$ in $\mathrm{Y}$-direction. This is mostly caused by the position error of the $\mathrm{C}$-axis average line at $B=0^{\circ},\left(\delta x_{C B}^{0}+\delta x_{B Y}^{0}\right.$, $\left.\delta y_{C B}^{0}\right)$.

- In Fig. 7(b), the bottom surface of each step is tilted toward both Xand Y-directions. This is mostly caused by the orientation error of the C-axis average line at $B=0^{\circ}$ to $\mathrm{X}$ - and Y-directions, $\left(\alpha_{C B}^{0}+\alpha_{B Y}^{0}\right.$, $\left.\beta_{C B}^{0}\right)$.

- Figure 8(a) shows the square with four side faces machined at $C_{j}=$ $0,90,180,270^{\circ}$ is smaller than the nominal geometry by about $40 \mu \mathrm{m}$. This is mostly attributable to the Z-position error of the B-axis average line, $\delta z_{B Y}^{0}$.

Then, location errors, shown in Table 1, are identified as shown in Fig. 9. Figure 10 shows position-dependent geometric errors of B-axis, shown in Table 2, identified from the measured geometry. While location errors in Fig.. 9 represent "average" position and orientation of rotary axes, Fig. 10 shows how the B-axis position and orientation vary with the B-axis angular position. 


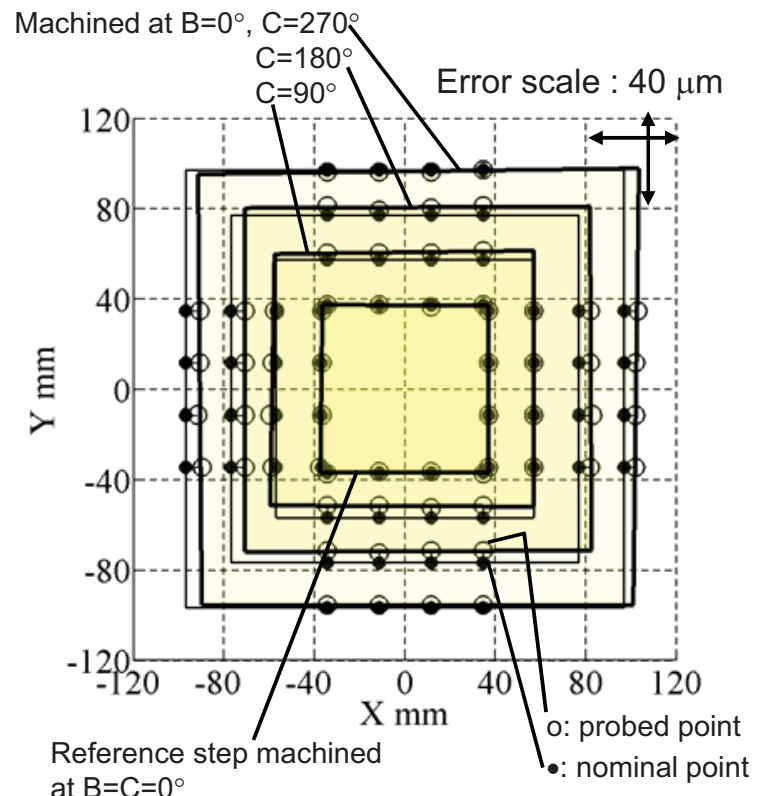

(a)

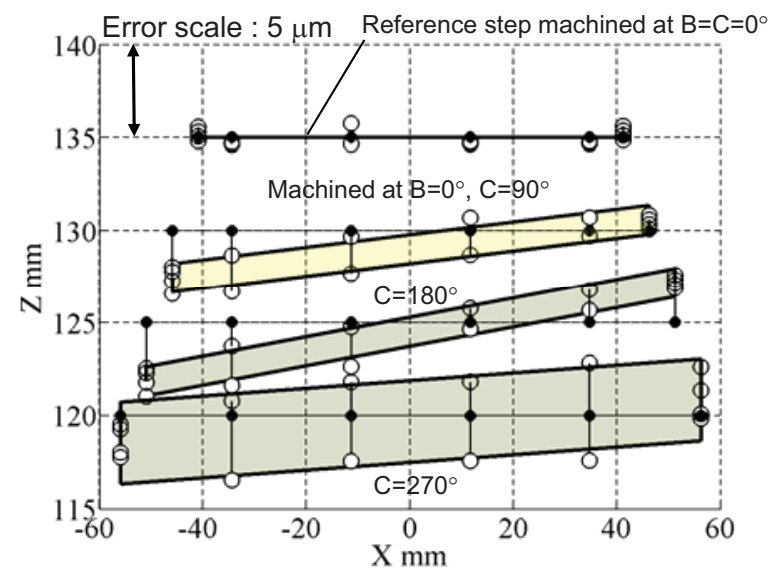

(b)

Figure 7: Measured geometry of the machined test piece (four steps machined at at $B_{i}=0^{\circ}$, and $C_{j}=0,90,180,270^{\circ}$ ). (a) projection onto XY plane, (b) projection onto XZ plane. 


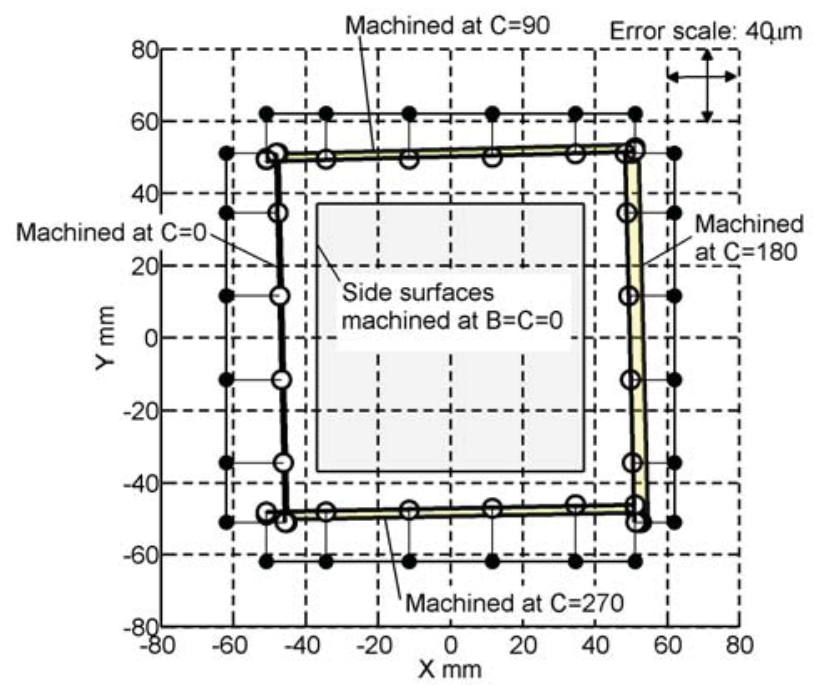

(a)

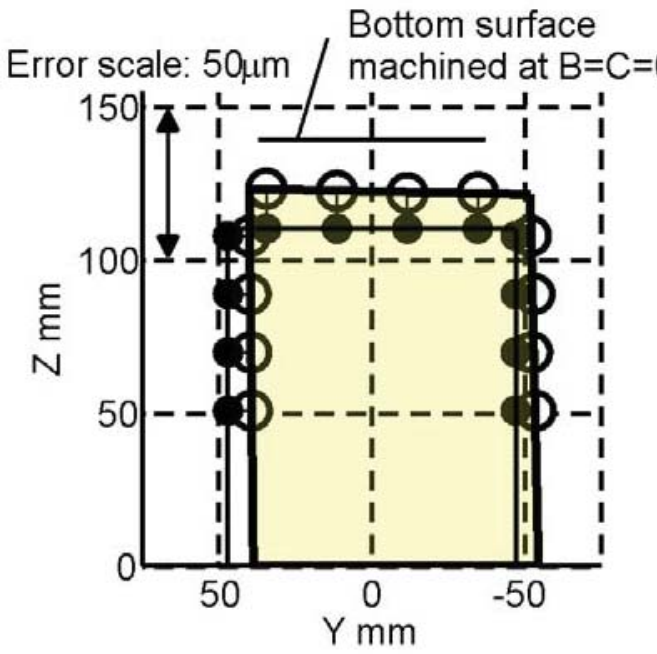

(b)

Figure 8: Measured geometry of the machined test piece. (a) Four steps on side faces machined at at $B_{i}=90^{\circ}$ and $C_{j}=0,90,180,270^{\circ}$, projected onto XY plane. (b) One of steps on side faces machined at at $B_{i}=90^{\circ}$ and $C_{j}=0^{\circ}$, projected onto YZ plane. 


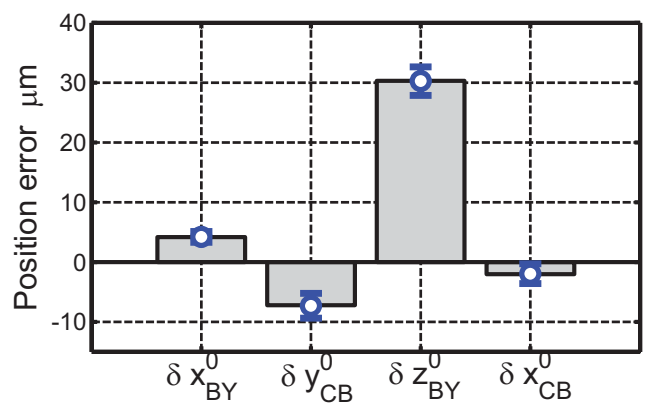

(a)

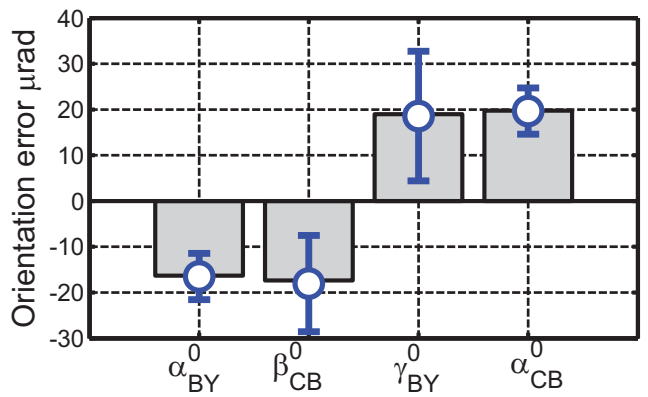

(b)

Figure 9: Position and orientation of B- and C-axis average lines (location errors, see Table 1) identified by the first machining test. Error bars represent the combined uncertainty $(k=1)$ calculated from uncertainty contributors in Table 4 (see Section 6). (a) Position errors. (b) Orientation errors. 


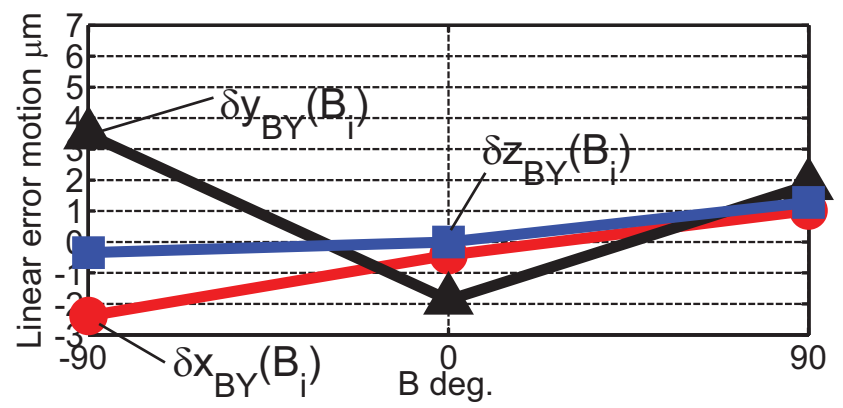

(a)

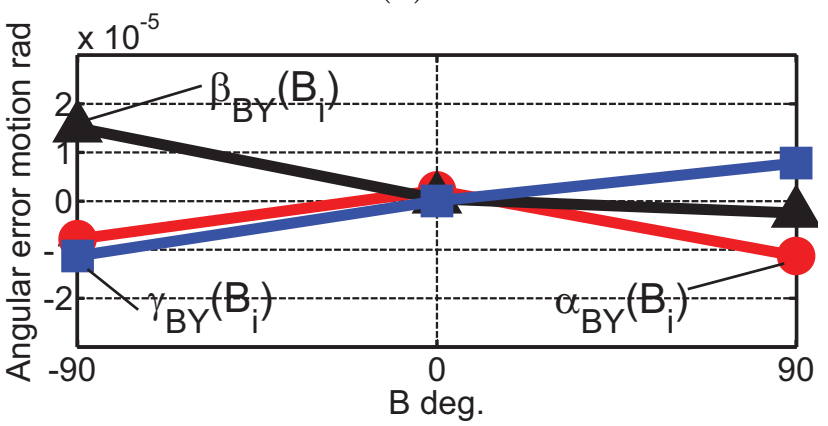

(b)

Figure 10: Position-dependent geometric errors (error motions) of B-axis (see Table 2) identified from measured geometry of the first test piece. (a) Position errors. (b) Orientation errors. 


\subsection{Comparison with non-cutting geometric error calibration}

Without any spindle-warm up, the probe-based error calibration scheme in Section 4.2 was performed. The total 192 points were probed at $B_{i}=$ $-90,0,90^{\circ}$, and $C_{j}=0,90,180,270^{\circ}$. The test took about 20 min. Figure 11 compares rotary axis location errors identified by the pre-machining probing test and the first machining test.

The largest difference (about $25 \mu \mathrm{m}$ ) can be observed in $\delta z_{B Y}^{0}$. The difference in $\delta x_{B Y}^{0}$ and $\delta y_{C B}^{0}$ is about $10 \mu \mathrm{m}$. Other location errors do not show significant difference. From the machine configuration in Fig. 1, it can be predicted that the spindle motor's heat would not significantly affect the position of rotary axes or their error motions. The test results suggest that the spindle heat mainly deforms the spindle-side machine structure toward the Z-direction. The displacement of linear axes changes the position of rotary axes in the machine coordinate system. The probing test indicates that the position of rotary axes was well calibrated in the "cold" condition, but the spindle rotation introduced significant errors. The present comparison clarifies the importance of calibrating these parameters in the condition thermally the same as actual machining processes. 


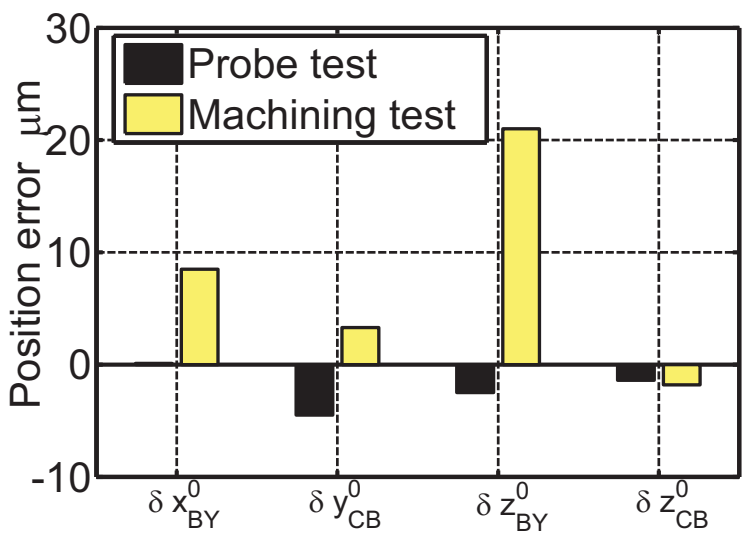

(a)

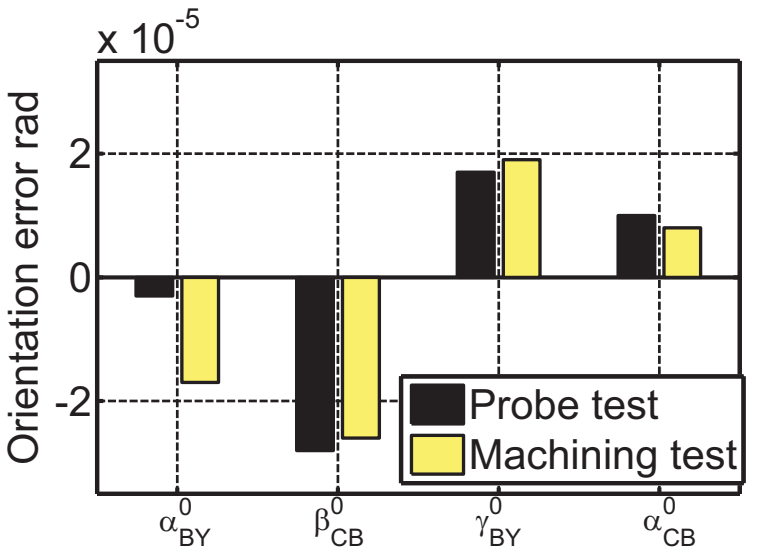

(b)

Figure 11: Location errors identified by the pre-machining probing test in the "cold" condition ("Probe test") and those by the first machining test ("Machining test"). (a) Position errors. (b) Orientation errors. 


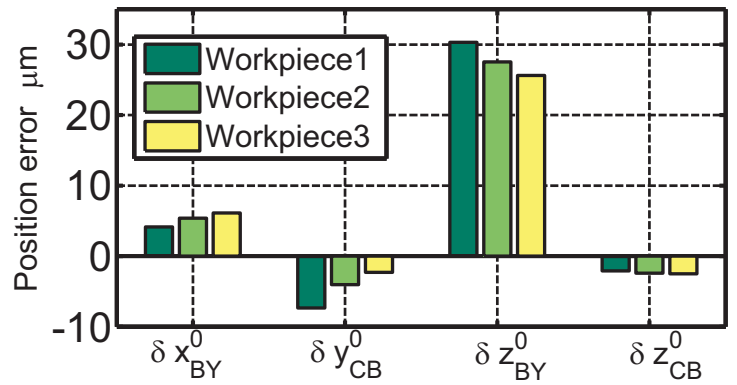

(a)

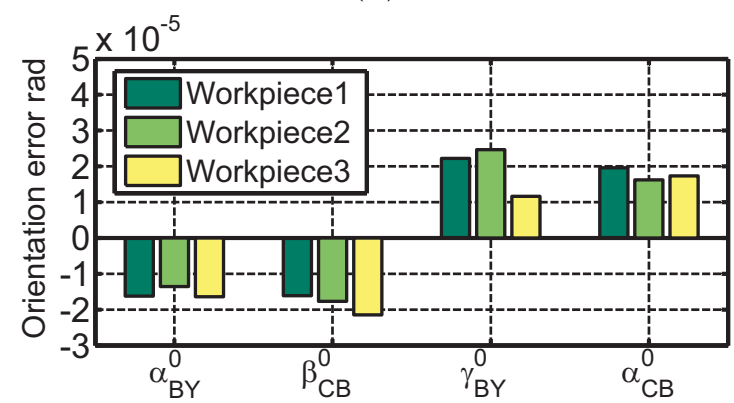

(b)

Figure 12: Location errors identified by three consequent machining tests.

\subsection{Changes in geometric errors with consequent machining tests}

Figure 12 shows location errors identified by the geometry of each of three finished test pieces. Notable gradual change can be observed in the Y-position of the $\mathrm{C}$-axis average line, $\delta y_{C B}^{0}$, and the Z-position of the B-axis average line, $\delta z_{B Y}^{0}$. Similarly as in Section 5.3, it indicates that the spindle's heat deforms the spindle-side machine structure mainly toward Z- and Ydirections. 


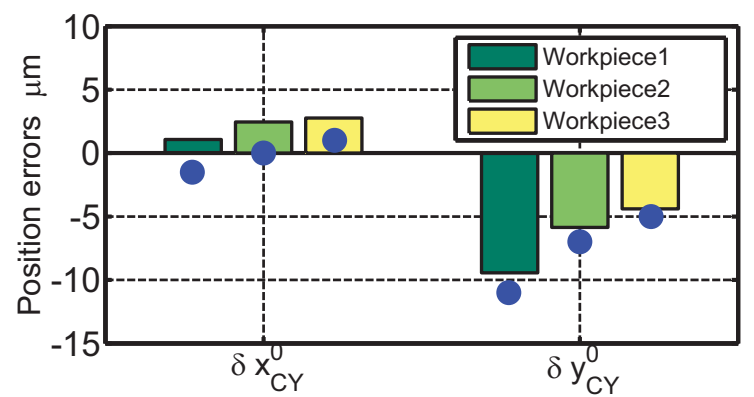

Figure 13: Comparison of C-axis position errors estimated from machined test pieces (bars) and those directly measured by using a dial gauge (dots $\bullet$ ).

\subsection{Validation of geometric errors identified by machining tests}

To validate location errors estimated from the machined test pieces, the position error of the C-axis average line, $\delta x_{C Y}=\delta x_{B Y}+\delta x_{C B}$ and $\delta y_{C Y}=$ $\delta y_{C B}$, were directly measured right after each test piece was finished. They were measured by using a dial gauge, attached to the spindle, and the topmost reference step of the test piece. Figure 13 compares the estimates and direct measurements. The validity of the estimates can be observed. The comparison with a larger set of geometric error parameters would be more difficult, since the direct measurement must be done without stopping the spindle for too long.

\section{Uncertainty analysis}

As was briefly discussed in Section 3.1, the algorithm presented in Section 3 assumes negligibly small error motions of linear axes. As is discussed 
in details in [35], linear axis error motions can be major uncertainty contributors for any "indirect" rotary axis error calibration schemes. This section assesses the uncertainty in identified geometric error parameters due to linear axis error motions. Other potential contributors, e.g. the uncertainty associated with the probing and the machining, are regarded relatively small.

Statistical uncertainty analysis based on the Monte Carlo simulation, analogous to the one presented in [35, 36], is applied. Table 4 shows assessed uncertainty contributors in linear axis error motions. The uncertainty contribution of each linear axis error motion is modelled as presented in [35, 36]. The values in Table 4 were assessed based on accuracy test results by the machine manufacturer, and partly by direct measurement of the machining center used in experiments.

In Fig. 9, error bars represent the combined uncertainty $(k=1)$ in each location error identified by the machining test. The contribution of linear axis error motions both on (1) the geometry of the finished test piece and (2) its on-the-machine measurement are considered. The uncertainty in identified position-dependent errors in Fig. 10 can be assessed similarly, but omitted here.

\section{Conclusion}

This paper proposes a machining test, where a simple square-shaped step is finished by a straight (radius) end mill at the given set of B- and C-axis angular positions. While our previous test proposed in [25] only identifies rotary axis location errors, the proposed test fully parameterizes the position 
Table 4: Uncertainty contributors in linear axis error motions (range for uniform distribution).

\begin{tabular}{c|l|l}
\hline Linear positioning errors, & Linear term & $4 \mu \mathrm{m} / 100 \mathrm{~mm}$ \\
$E_{X X}, E_{Y Y}, E_{Z Z}$ & Periodic term & $0.5 \mu \mathrm{m}$ \\
Straightness errors, $E_{Y X}, E_{Z X}$, & Periodic term & $0.5 \mu \mathrm{m}$ \\
$E_{X Y}, E_{Z Y}, E_{X Z}, E_{Y Z}$ & Non-periodic term & $2 \mu \mathrm{m}$ \\
Angular errors, $E_{A X}$, & Periodic term & $2.5 \times 10^{-5} \mathrm{rad}$ \\
$E_{B X}, E_{A Y}, E_{B Y}, E_{C Y}$ & & $1.5 \times 10^{-5} \mathrm{rad}$ \\
Squareness errors, & & \\
$E_{C(0 X) Y}, E_{B(0 X) Z}, E_{A(0 Y) Z}$ & & (normal distribution) \\
Non-systematic error & $\sigma=0.35 \mu \mathrm{m}$ \\
\hline
\end{tabular}

and the orientation of two rotary axes at each index angle.

By repeating the proposed machining test, the change in geometric error parameters can be observed, which is mostly caused by thermal deformation induced by spindle rotation or environmental change. In our experience, thermally-induced variation in rotary axis location errors can be one of the most critical error factors for five-axis machining. The accuracy tests for machine tools, e.g. static tests described in [1], are sometimes done when the machine is "cold" in, e.g., machine assembly or inspection by machine tool builders. The proposed machining test is effective to evaluate geometric errors when the machine is in normal operating conditions.

As a final remark, ISO/DIS 10791-7.2 [19], currently under revision process in ISO/TC39/SC2, contains a new five-axis machining test (M4). The test is similar but different; at $B=0^{\circ}$, a single square is machined with each side face end-milled at $C=0,90,180,270^{\circ}$. Then, at $B=90^{\circ}$ and $-90^{\circ}$, 
a square is machined with each side face face-milled at $C=0,90,180,270^{\circ}$.

Clearly, this test does not enable a user to identify the full set of positiondependent geometric errors of rotary axes. More studies would be needed to understand what geometric errors affect test results.

\section{Acknowledgement}

The machining center used in present experiments is loaned by the Machine Tool Technologies Research Foundation (MTTRF) via Equipment on Loan Award Program.

\section{References}

[1] ISO/DIS 10791-1:2012, Test conditions for machining centres - Part 1: Geometric tests for machines with horizontal spindle (horizontal Z-axis).

[2] ISO 230-7:2006, Test code for machine tools - Part 7: Geometric accuracy of axes of rotation.

[3] H. Schwenke, W. Knapp, H. Haitjema, A. Weckenmann, R. Schmitt, F. Delbressine, Geometric error measurement and compensation of machines -An update, CIRP Annals - Manufacturing Technology 57 (2) (2008) 560-575.

[4] S. Ibaraki, W.Knapp, Indirect Measurement of Volumetric Accuracy for Three-axis and Five-axis Machine Tools: A Review, Int' l J. of Automation Technology, 6 (2) (2012). 
[5] Y. Kakino, Y. Ihara, H. Sato, H. Otsubo, "A Study on the motion accuracy of NC machine tools (7th report) -Measurement of motion accuracy of 5-axis machine by DBB tests-," J. of Japan Society for Precision Engineering, 60-5, pp.718-723, 1994 (in Japanese).

[6] Y. Abbaszaheh-Mir, J. R. R. Mayer, G. Clotier, C. Fortin, Theory and simulation for the identification of the link geometric errors for a fiveaxis machine tool using a telescoping magnetic ball-bar, International Journal of Production Research 40 (18) (2002) 4781-4797.

[7] M. Tsutsumi, A. Saito, Identification and compensation of systematic deviations particular to 5-axis machining centers, International Journal of Machine Tools and Manufacture 43 (2003) 771-780.

[8] ISO/FDIS 10791-6:2014 Test conditions for machining centres - Part 6: Accuracy of speeds and interpolations.

[9] S. Weikert, R-Test, a New Device for Accuracy Measurements on Five Axis Machine Tools, Annals of the CIRP 53 (1) (2004) 429-432.

[10] B. Bringmann, W. Knapp, Model-based 'Chase-the-Ball' calibration of a 5-axis machining center, Annals of the CIRP 55 (1) (2006) 531-534.

[11] T. Erkan, J. R. R. Mayer, and Y. Dupont, Volumetric distortion assessment of a five-axis machine by probing a 3D reconfigurable uncalibrated master ball artefact, Precision Engineering 35 (2011) 116-125.

[12] T. Matsushita and T. Oki, Identification of Geometric Errors in Fiveaxis Controlled Machine Tool with Touch Trigger Probe, Proc. of the 2010 Spring JSPE Semiannual meeting (2010) 1105-1106 (in Japanese). 
[13] AxiSet ${ }^{\mathrm{TM}}$ Check-Up by Renishaw, http://www.renishaw.com/

[14] ISO 230-3:2007, Test code for machine tools - Part 3: Determination of thermal effects.

[15] C. Hong, S. Ibaraki, Observation of Thermal Influence on Error Motions of Rotary Axes on a Five-Axis Machine Tool by Static R-Test, Int'l J. of Automation Technology 6-2 (2012) 196-204.

[16] M. Gebhardt, P. Cube, W. Knapp, K. Wegener, Measurement set-ups and -cycles for thermal characterization of axes of rotation of 5-axis machine tools, Proc. the 12th euspen Int'l Conf. (2012).

[17] M. Gebhardt, S. Capparelli, M. Ess, W. Knap, K. Wegener, Physical and phenomenological simulation models for the thermal compensation of rotary axes of machine tools, Proc. the 13th euspen Int'l Conf. (2013).

[18] NAS 979:1969, Uniform cutting test - NAS series, metal cutting equipment specifications; 34-37.

[19] ISO/DIS 10791-7.2:2014, Test Conditions for Machining Centres - Part 7: Accuracy of a Finished Test Piece.

[20] C. Hong, S. Ibaraki, A. Matsubara. Influence of position-dependent geometric errors of rotary axes on a machining test of cone frustum by five-axis machine tools. Precision Engineering 35-1 (2011) 1-11.

[21] S. Bossoni, Geometric and Dynamic Evaluation and Optimization of Machining Centers, Ph.D. dissertation, ETH Zurich (2009). 
[22] K. Ohta, Z. Li, M. Tsutsumi, Proposal of a Machining Test of Five-Axis Machining Centers Using a Truncated Square Pyramid, Key Engineering Materials 523-524 (2012) 475-480.

[23] H. Takeshima, Y. Ihara, Finished Test Piece Example for Five-axis Machining Centers, Proc. Int'l Conf. on Leading Edge Manufacturing in 21st century (2009) 123-126.

[24] S. Zhu, G. Ding, S. Qin, J. Lei, L. Zhuang, K. Yan, Integrated geometric error modeling, identification and compensation of CNC machine tools, Int'l J. Machine tools and Manufacture 52 (2012) 24-29.

[25] S. Ibaraki, M. Sawada, A. Matsubara, T. Matsushita, Machining tests to identify kinematic errors on five-axis machine tools, Precision Engineering 34 (3) (2010), 387-398.

[26] Y. Morimoto, K. Nakato, M. Gontan, Accuracy Evaluation of 5-Axis Machining Center Based on Measurements of Machined Workpiece Evaluation of Accuracy of 5-Axis Controlled Machining Center, Int'l J. Automation Technology 6(5) (2012) 675-681.

[27] ISO 230-1:2012, Test code for machine tools - Part 1: Geometric accuracy of machines operating under no-load or quasi-static conditions.

[28] I. Inasaki, K. Kishinami, S. Sakamoto, N. Sugimura, T. Takeuchi, F. Tanaka, Shaper generation theory of machine tools - its basis and applications, Yokendo, Tokyo, 1997 (in Japanese).

[29] D.N. Reshetov, V.T. Portman, Accuracy of Machine Tools, ASME Press, 1988. 
[30] Hocken, R.J., Simpson, J.A., Borchardt, B., Lazar, J., Reeve, C., Stein, P.,1977, Three dimensional metrology, Annals of CIRP, 26(1), pp.403408.

[31] S. Ibaraki, C. Oyama, H. Otsubo, Construction of an error map of rotary axes on a five-axis machining center by static R-test, International Journal of Machine Tools and Manufacture 51(3) (2011) 190-200.

[32] S. Ibaraki, T. Iritani, T. Matsushita, Error map construction for rotary axes on five-axis machine tools by on-the-machine measurement using a touch-trigger probe, International Journal of Machine Tools and Manufacture 68 (2013) 21-29.

[33] S. Ibaraki, Y. Ota, Error Calibration for Five-Axis Machine Tools by Onthe-Machine Measurement Using a Touch-Trigger Probe. International Journal of Automation Technology 8(1) (2014) 20-27.

[34] ISO 26303:2012, Machine tools - Short-term capability evaluation of machining processes on metal-cutting machine tools.

[35] B. Bringmann, W. Knapp, Machine tool calibration: Geometric test uncertainty depends on machine tool performance, Precision Engineering, 33 (2009) 524-529.

[36] B. Bringmann, J. P. Besuchet, L. Rohr, Systematic evaluation of calibration methods, CIRP Annals Manufacturing Technology 57 (2008) $529-532$. 